of trajectories of extraversion and neuroticism personality traits in individuals who did and did not receive a dementia diagnosis.

This study used data from the OCTO-Twin Study, Longitudinal Aging Study Amsterdam, Swedish Adoption Twin Study of Aging, and Einstein Aging Study. For each dataset, a series of latent growth curve models were run examining each personality trait, first including a subsample of individuals eventually diagnosed with dementia and timeto-dementia metric, and second including the entire dataset, dementia diagnosis as a variable, and time-in-study metric.

Controlling for sex, age, education, depressive symptoms, and the interaction between age and education, the first series of analyses revealed a consistent pattern of personality change preceding dementia diagnosis across datasets, specifically linear increases in neuroticism and stability in extraversion. The second series of analyses revealed a less stable pattern of results: dementia diagnosis was only a significant predictor of neuroticism trajectories in some datasets. These findings will be discussed.

Identification of early indicators of dementia, specifically how personality changes differ for healthy individuals compared to individuals eventually diagnosed with dementia, may aid in early care strategies and facilitate development of screening assessments.

\section{PERSONALITY TRAITS PREDICT DIETARY HABITS IN MIDDLE-TO-OLDER ADULTS}

S. Weston ${ }^{1,2}$, G. Edmonds 3 , S. Hampson ${ }^{3}$, P. Hill ${ }^{1}, 1$. Psychology, Washington University in St. Louis, Evanston, Illinois, 2. Northwestern University, Evanston, Illinois, 3. Oregon Research Institute, Eugene, Oregon

Personality traits predict dietary habits in middle-to-older adults.

Personality traits are consistently associated with health behaviors, but little research has examined the role of traits on eating habits among middle-to-older adults. Importantly, food choices are constrained by financial resources or availability of healthy options, suggesting the need to test differential associations across SES. We examined the associations between traits and dietary habits, whether healthy eating predicted health at age 60, and if SES moderated these relationships. We used a sample of 665 middle-to-older adults from the Hawaii Personality and Health Cohort. Participants completed personality and eating questionnaires at age 44 and reported health at age 60 . Dietary items were consolidated using factor analysis, which resulted in two factors: healthy and unhealthy foods. Eating healthy foods was associated with higher levels of agreeableness $(r=.11)$, conscientiousness (.14), emotional stability (.14) and openness (.19) and predicted better self-rated health (.21) and lower BMI (-.19). Eating unhealthy foods was associated with lower levels of agreeableness $(\mathrm{r}=-.11)$, conscientiousness $(-.12)$, emotional stability (-.09) and openness (-.14) and predicted lower selfrated health (-.13). Unhealthy food consumption did not predict BMI. Surprisingly, these results were not moderated by SES. Overall, we conclude that personality traits have a consistent relationship with dietary habits across levels of SES, and thus the use of traits to predict and understand eating choices can be applied consistently across patients, regardless of financial resources. Moreover, these associations between traits and eating choices may consequences for older-adult health.

\section{STRESS, PHYSICAL ACTIVITY, AND AGING: COORDINATED ANALYSES OF TWO LONGITUDINAL DATA SETS}

R. Vendittelli, Psychology, University of Victoria, Saanich, British Columbia, Canada

Although modest declines in cognition are considered normative, it is becoming increasingly evident that severe decline and dementia are not inevitable outcomes caused by age. Person-specific variables such as physical activity (PA) and stress are two modifiable lifestyle factors that have been demonstrated to be associated with poorer cognitive outcomes. To date, there are no studies examining both the between-person (BP; or person-mean levels) and within person (WP; or occasion specific fluctuation relative to one's expected trajectory) effects of PA and stress on cognitive abilities. Data from two longitudinal data sets were analyzed in a coordinated manner. Specifically, utilizing comparable variables, data were fit to the same models with processing speed, memory and MMSE as separate outcome variables. Higher average PA was associated with less decline in processing speed, working memory and MMSE scores in one of the two data sets. Interestingly, the WP effects of PA were associated with better cognitive outcomes in all models except memory performance in one of the studies. Unexpectedly, the WP effects of stress were associated with higher processing speed and working memory performance in one of the studies, while the BP effects were non-significant across both studies and outcome variables. Findings indicate that higher average PA, as well as individual specific fluctuations in activity can positively influence cognition. Moreover, proximal stress seems to enhance cognition. These findings corroborate literature implicating activity in cognitive functioning, and are nuanced in demonstrating that more proximal indicators of activity level and stress are associated with cognitive performance.

\section{THE IMPORTANCE OF SUFFICIENT CHALLENGES TO POSITIVE EMOTIONS AMONG OLDER ADULTS}

D. Tse, J. Nakamura, M. Csikszentmihalyi, Quality of

Life Research Center, Claremont Graduate University,

Claremont, California

Flow theory postulates that people enjoy the optimal experience when challenge level of an activity matches their skill level. However, little research has extended the theory to examine the effect of challenge-skill balance on older adults' emotional experiences. This recently completed study investigated whether older adults experienced more frequent high arousal positive(HAP) and low arousal positive(LAP) emotions in challenge-skill balanced activities. 104 younger(Mage=25.8, SD=2.76) and 93 older adults (Mage=65.0, SD=4.78) identified underchallenging(challenge-lower-than-skill), challenge-skill balanced, and overchallenging(challenge-higher-than-skill) activities that they had chosen to do in the past, and reported their typical emotions in each activity. Results were analyzed by repeated measures ANOVA and paired comparison t-tests. Consistent with flow theory, both younger and older adults reported more frequent HAP and LAP emotions 